Z. Klin. Chem. Klin. Biochem.

12. Jg. 1974, S. $33-38$

\title{
Untersuchungen zur Frage einer Aminosäuren-Imbalance unter den Bedingungen einer experimentellen Hypertyrosinämie bei der Ratte
}

\author{
Von E. Schaumlöffel und G. Fekete ${ }^{1}$ ). \\ Aus der Klinik und Poliklinik für Nuklearmedizin im Radiologiezentrum und der Kinderklinik und Poliklinik \\ der Philipps-Universität Marburg (Lahn)
}

(Eingegangen am 2. Juli/25. Oktober 1973)

Herrn Prof. Dr. H. Kühn zum 60. Geburtstag gewidmet

Bei 5 Wochen alten Ratten (60 g Körpergewicht) wurde ein intraperitoneales Depot von $5 \mathrm{~g}\left(3,5-{ }^{3} \mathrm{H}\right] L$-Tyrosin/kg Körpergewicht gesetzt. 2 Stunden nach i.v. Applikation von $\left[1-{ }^{14} \mathrm{C}\right] L$-Leucin wurden die Tiere getötet und in Serum, Gehirn, Leber, Lunge, Milz und Nieren die Konzentrationen an freien Aminosäuren und die Utilisation der markierten Pools in der Proteinsynthese analysiert. In Serum, Hirn und Leber wurden die Konzentrationen an freien Aminosäuren säulenchromatographisch bestimmt. Bedingt durch eine hohe Abbaurate zu Stoffwechselendprodukten (42\% der Dosis) konnte sich die hohe Tyrosin-Dosis nicht im Sinne einer Hypertyrosinämie auswirken, d. h. es wurde weder eine Hemmung des Transportes essentieller Aminosäuren in die Zellen noch eine Störung der Protein-Synthese festgestellt. Auch die relativ hohe Konzentration an intermediären Stoffwechselprodukten des Tyrosins in den Geweben ließ keinen negativen Einfluß auf die Stoffwechselvorgänge erkennen.

The question of amino acid imbalance under the conditions of experimental hypertyrosinaemia in the rat

Rats (age 5 weeks, weight $60 \mathrm{~g}$ ) were injected intraperitoneally with $5 \mathrm{~g}\left[3,5-{ }^{3} \mathrm{H}\right] L$-tyrosine per kg body weight. 2 Hours after the intravenous application of $\left[1-{ }^{14} \mathrm{C}\right] L$-leucine, the animals were killed, and the concentration of free amino acids and the utilization of the labelled pool for protein synthesis were determined in the serum, brain, liver, lung, spleen and kidneys. In the serum, brain and liver, the concentrations of free amino acids were determined by column chromatography. Owing to a rapid degradation to metabolic end products, the high dose of tyrosine did not result in a hypertyrosinaemia, $i$. e., there was neither an inhibition of the transport of essential amino acids into the cells, nor an interference in protein synthesis. The relatively high tissue concentration of tyrosine metabolites had no recognizable negative effect on tissue metabolism.

Die seit langem als Adaptationsstörung besonders der Frühgeborenen bekannte temporäre Hypertyrosinämie mit einer vorübergehenden Erhöhung des Serumspiegels bis zum 40 fachen der Norm ( $15 \mathrm{mg} / \mathrm{l})$ tritt ohne sicher erkennbare klinische Symptome auf (1). Sie wird mit einer Eiweiß-Überernährung bei noch nicht ausgereiften Enzymsystemen des Frühgeborenen in Zusaminenhang gebracht (2). In jüngster Zeit stellten Menkes et al $(3,4)$ bei Kontrolluntersuchungen ehemals hypertyrosinämischer Frühgeborener im Lebensalter von 7 und 8 Jahren intellektuelle Funktionsstörungen fest. Neben einem direkten neurotoxischen Effekt des überhöhten Tyrosinspiegels werden diese neurologischen „Spätschäden“ wie bei anderen Hyperaminoacidämien als unspezifisches Leitsymptom gesehen. Als Ursache werden auf Grund der Ähnlichkeit der Symptomatik mit anderen erblichen Aminosäure-Stoffwechselstörungen - z. B. der Phenylketonurie - auch bei der temporären Hypertyrosinämie die durch kompetitive Hemmung des Transportes essentieller Aminosäuren nachgewiesenen Konzentrationsungleichgewichte (Aminosäuren-Imbalance) in ihrer schädigenden Auswirkung auf die Proteinsynthese in einem kritischen Stadium der Hirnentwicklung diskutiert $(5,6,7)$. Diese Hypothese scheint auch durch experimentelle Befunde an der Ratte gestützt zu wer- den (8), die jedoch von anderen Autoren nicht bestätigt werden konnten $(9,10)$. Bei in vitro- und in vivo-Untersuchungen konnte dagegen festgestellt werden, daß die Permeation von Tyrosin in die Hirnzellen durch überhöhte Konzentrationen anderer aromatischer und langkettiger aliphatischer Aminosäuren gestört $\mathrm{zu}$ werden scheint $(11,12,13)$.

In der vorliegenden Studie haben wir nach Setzen eines hohen intraperitonealen Depots an $\left[{ }^{3} \mathrm{H}\right]$ Tyrosin $(5 \mathrm{~g} / \mathrm{kg}$ Körpergewicht) bei der 5 Wochen alten Ratte nach anschließender Injektion von trägerfreiem $\left[{ }^{14} \mathrm{C}\right]$ Leucin über die Radioaktivitätsbestimmung zu einem fixierten Zeitpunkt die Konzentration an freiem Tyrosin und Leucin und die in Organproteine eingebauten Anteile dieser Aminosäuren bestimmt. Durch Erfassung der Bildungsrate an Tritiumwasser war eine Aussage über die Verstoffwechselung des Tyrosins zu Endprodukten möglich. Die Bestimmung der Konzentrationen an freien Aminosäuren in Serum, Gehirn und Leber mit Hilfe der säulenchromatographischen Analyse erlaubte eine Aussage über mögliche Aminosäure-Imbalancen in Serum und Organen.

1) 2. Universitäts-Kinderklinik, Budapest/Ungarn 


\section{Material und Methodik}

\section{Chemikalien}

Alle im folgenden aufgeführten Chemikalien besaßen den . Reinheitsgrad p. a. bzw. für biochemische Zwecke ( $L$-Tyrosin) und wurden von der Firma Merck, Darmstadt, bezogen.

Die speziellen Szintillationschemikalien Permablend III, Instagel, Hyamin-Hydroxid sowie ${ }^{3} \mathrm{H}$ - und ${ }^{14} \mathrm{C}$-markiertes Toluol lieferte die Firma Packard, Frankfurt/Main.

Von der Firma Amersham-Buchler, Braunschweig, bezogen wir $\left[3,5-{ }^{3} \mathrm{H}\right] L$-Tyrosin mit einer spez. Akt. von $233 \mathrm{mCi} / \mathrm{mg}$ und $\left[1{ }^{14} \mathrm{C}\right] L$-Leucin mit einer spez. Akt. von $0,56 \mathrm{mCi} / \mathrm{mg}$.

\section{Tierexperimente}

Die Untersuchungen wurden mit 2 Gruppen von je 10 weiblichen Wistar-Ratten (Alter 5 Wochen; Durchschnittsgewicht $60 \mathrm{~g}$ ) durchgeführt, die bis zu Versuchsbeginn bei normaler Haltung und Fütterung (Altromin-Standardfutter und Wasser ad lib.) gehalten worden waren. Die Tiere beider Gruppen erhielten in 90minütigen Abständen 3 intraperitoneale Injektionen von je $16,67 \mu \mathrm{Ci}\left[3,5-{ }^{3} \mathrm{H}\right] L$-Tyrosin bzw. einer $\mathrm{Ge}$ samtaktivitätsdosis von $50 \mu \mathrm{Ci}^{3} \mathrm{H}$. In der Versuchsgruppe (V-Gruppe) wurde die spez. Akt. des Tyrosins mit inaktivem $L$-Tyrosin auf $0,167 \mu \mathrm{Ci} / \mathrm{mg}$ eingestellt, so daß bei einer Einzeldosis von $100 \mathrm{mg}$ in wäßr. Suspension von $1,0 \mathrm{ml}$ appliziert, die Gesamtdosis $300 \mathrm{mg} /$ Tier bzw. $5 \mathrm{~g} / \mathrm{kg} \mathrm{Körpergewicht}$ betrug. In der Kontrollgruppe (K-Gruppe) lag die Gesamtdosis von $L$-Tyrosin (3 Applikationen in je 1,0 ml physiol. $\mathrm{NaCl}$ ) bei $0,21 \mu \mathrm{g} /$ Tier.:

2 Stunden nach der letzten Applikation von $\left[{ }^{3} \mathrm{H}\right] L$-Tyrosin injizierten wir jedem Tier der beiden Grtippen in die freigelegte vena femoralis $20 \mu \mathrm{Ci}\left[1-{ }^{14} \mathrm{C}\right] L$-Leucin (spez. Akt. $0,56 \mathrm{mCi} / \mathrm{mg}$ ) in $0,2 \mathrm{ml}$ physiol. $\mathrm{NaCl}$ (ca. $35 \mu \mathrm{g} \mathrm{L}$-Leucin/Tier).

Während des Versuches starb eins der Tiere der V-Gruppe ohne erkennbare Ursache. Die übrigen Tiere zeigten normales Verhalten.

2 Stunden nach der Leucin-Injektion wurden die Tiere nach Herzpunktion in Äthernarkose durch Dekapitieren getötet und die Organe Gehirn, Leber, Lunge, Milz und Nieren entnommen. Die Organe wurden vor der weiteren Aufbereitung in kalter, $20 \%$ Methanol enthaltender physiol. $\mathrm{NaCl}$ Lösung gewaschen.

\section{Probenaufbereitung żur Radioaktivitätsbestimmung}

Die Organe jedes einzelnen Tieres wurden mit einem Homogenisator nach Potter und Elvehjem (Firma B. Braun, Melsungen) bzw. mit einem Ultra-Sonator (Firma Schöller \& Co, Frankfurt/Main, Modell TG 125) homogenisiert. Aliquote Teile der frischen Organhomogenate (2 Parallelen) wurden in Zentrifugengläser eingewogen und die Proteine durch Zusatz von $3,0 \mathrm{ml} \mathrm{HClO} 4(0,7 \mathrm{~mol} / 1)$ in der Kälte gefällt und die Rückstände zweimal mit $\mathrm{HClO}_{4}$ bzw. physiol. $\mathrm{NaCl}$ gewaschen; die drei nach Zentrifugieren und Dekantiereı gewonnunen Überstände wurden vereinigt und 1,0 ml der Extrakte (2 Parallelen) wurde gefriergetrocknet (Firma Leybold, Köln, Modell GT 2), mit $0,5 \mathrm{ml}$ Hyamin-Hydroxyd aufgenommen und $\mathrm{mit} 15 \mathrm{ml}$ Szintillationslösung $(5,5 \mathrm{~g}$ Permablend III $+200 \mathrm{ml}$ Äthanol + Toluol ad $1000 \mathrm{ml}$ ) versetzt.

Von den gewogenen Perchlorsäure-Präzipitaten wurden aliquote Teile (2 Parallelen von 50-100 mg Feuchtgewicht) auf Filterpapier eingewogen und nach Gefriertrocknung in einem Verbrennungsautomaten (Firma Packard, Modell 305) verbrannt. ${ }^{3} \mathrm{H}_{2} \mathrm{O}$ wurde in $10 \mathrm{ml}$ Instagel aufgenommen und ${ }^{14} \mathrm{CO}_{2}$ nach Absorption in 3,0 ml Äthanolamin und Nachwaschen mit 7,0 ml Methanol mit $5 \mathrm{ml}$ Szintillationslösung II (15 g Permablend III in $1000 \mathrm{ml}$ Toluol) versetzt.

$\mathrm{Je} 0,1 \mathrm{ml}$ Serum (2 Parallelen) wurden vor und nach Gefriertrocknung in $0,5 \mathrm{ml}$ Hyamin aufgenommen und nach Zugabe von $15 \mathrm{ml}$ Szintillationslösung I zur Aktivitätsbestimmung gebracht. Aus der Differenz der ${ }^{3} \mathrm{H}$-Radioaktivität vor und nach Gefriertrocknung wurde der auf ${ }^{3} \mathrm{H}_{2} \mathrm{O}$ entfallende Aktivitätsanteil errechnet.

Die Bestimmung an freiem Tyrosin und Leucin über die ${ }^{3} \mathrm{H}$ und ${ }^{14} \mathrm{C}$-Aktivität mußte wegen zu geringer Serummengen in den gepoolten Seren der Tiere beider Gruppen erfolgen. 1,0 ml der beiden Serumpools (2 Parallelen) wurden durch Zugabe von $3,0 \mathrm{ml}$ Äthanol enteiweißt und die Proteine zweimal mit physiol. $\mathrm{NaCl}$ gewaschen. Die nach Zentrifugieren und Dekantieren vereinigten Überstände wurden, wie bei den Organextrakten beschrieben, zur Radioaktivitätsbestimmung aufbereitet.

\section{Radioaktivitätsbestim mung}

Die ${ }^{3} \mathrm{H}$ - und ${ }^{14} \mathrm{C}$-Aktivitätsbestimmungen (Einfach- und Doppelmarkierung) erfolgten in einem 3-Kanal-Flüssigszintillationsspektrometer (Firma Packard, Modell 3380) mit automatischer Korrektur der Löscheffekte über das Kanalverhältnis des externen Standards $\left({ }^{266} \mathrm{Ra} /{ }^{243} \mathrm{Am}\right)$ und Datenausgabe in absoluter Aktivität (Zerfälle/min). Zur Korrektur der entsprechenden Quench-Bereiche wurde das Gerät für Einfach- und Doppelmarkierung mit internem Standard $\left({ }^{3} \mathrm{H}\right.$ - und ${ }^{14} \mathrm{C}$-Toluol $)$ geeicht.

\section{Auswertung der Ergebnisse}

Aus den Ergebnissen der Radioaktivitätsbestimmungen (Mittelwerte der parallelen Ansätze) in Serum- und Organproteinen sowie den Überständen nach Proteinfällung errechneten wir die spezifischen Aktivitäten, beżogen auf g Frischgewicht. Um unterschiedliche Körpergewichte bei gleicher Dosierung auszugleichen, berechneten wir die relativen spezifischen Aktivitäten (rel. spez. Akt.) in \% nach der Formel

$$
=\frac{\text { speż. Akt. } \cdot \text { g Körpergewicht } \cdot 100}{\text { Aktivitätsdosis }}
$$

$100 \%$ rel. spez. Akt. entsprechen somit der pro $\mathrm{g}$ Tier applizierten Dosis an ${ }^{3} \mathrm{H}$ - bzw. ${ }^{14} \mathrm{C}$ - Aktivität.

Die Konzentrationen an Tyrosin in $\mu \mathrm{mol} / \mathrm{g}$ berechneten wir in der V-Gruppe aus der spez. Akt. der applizierten Aminosäure $(0,167 \mu \mathrm{Ci} / \mathrm{mg})$. Danach entsprachen $100 \%$ rel. spez. Akt. $5 \mathrm{mg}$ Tyrosin/g Tier. Bei gleicher Aktivitätsdosis in der K-Gruppe war jedoch die Dosis an Tyrosin auf Grund der spez. Akt. von $233 \mathrm{mCi} / \mathrm{mg}$ so gering $(0,21 \mu \mathrm{g}$ Tyrosin/Tier $)$, daß diese Dosis gegenüber der Konzentration an freiem Tyrosin im Organismus der Tiere vernachlässigbar gering war. Wir berechneten daher die Dosis an Tyrosin auf der Basis des Serumspiegels $(12,5 \mu \mathrm{g} / \mathrm{g})$. Um den turnover des Tyrosins im Versuchszeitraum zu berücksichtigen, bezogen wir unsere Berechnungen auf das Doppelte dieser Serumkonzentration. Damit entsprachen $100 \%$ rel. spez. Akt. $25 \mu \mathrm{g} / \mathrm{g}$ Tier.

$\mathrm{Da}$ auch $\left[1-{ }^{14} \mathrm{C}\right]$ Leucin praktisch trägerfrei appliziert wurde, $\left(20 \mu \mathrm{Ci}{ }^{14} \mathrm{C}\right.$ bzw. $35 \mu \mathrm{g}$ Leucin/Tier) setzten wir die pro $\mathrm{g}$ Tier applizierte Aktivitätsdosis der Serumkonzentration an freiem Leucin gleich, so daß $100 \%$ rel. spez. Akt. $20 \mu \mathrm{g} \mathrm{Leucin/g}$ Tier entsprachen.

Zur Berechnung der Abbaurate von Tyrosin zu Stoffwechselendprodukten ermittelten wir die rel. spez. Akt. (\%) an ${ }^{3} \mathrm{H}_{2} \mathrm{O}$ im Serum und berechneten unter Zugrundelegen eines Körperwasseranteils von $60 \%$ des Körpergewichtes die Abbaurate in \% der applizierten Dosis.

Aus den jeweiligen Einzelwerten der 9 bzw. 10 Tiere beider Gruppen errechneten wir die Mittelwerte und die Standardabweichungen der Einzelwerte. Zur Absicherung der Unterschiede entsprechender Ergebnisse beider Gruppen wendeten wir den inhomogenen t-Test nach Student an.

\section{Mikrochemische Aminosäurenanaly se}

In Serum, Gehirn und Leber führten wir bei parallelen Ansätzen die Bestimmung der Konzentrationen an freien Aminosäuren durch. Von den gepoolten Seren beider Gruppen wurden in $0,1 \mathrm{ml}$ nach Versetzen mit $0,1 \mathrm{ml}$ Glucose-Phosphat-Puffer $(44,55 \mathrm{~g} / 1$ Glucose in $6,66 \mathrm{mmol} / 1$ Phosphatpuffer $\mathrm{pH} \mathrm{7,4)}$ und $0,1 \mathrm{ml} \mathrm{HClO}$ ( $(2,0 \mathrm{~mol} / 1)$ die Proteine gefällt und abzentrifugiert. Der Überstand wurde mit $\mathrm{KOH}(5,0 \mathrm{~mol} / \mathrm{l})$ auf $\mathrm{pH} 5,0$ eingestellt. Nach Abzentrifugieren des in der Kälte gefällten $\mathrm{KClO}_{4}$ wurden $0,2 \mathrm{ml}$ des Überstandes zur säulenchromatographischen Analyse aufgetragen. Die $\mathrm{HClO}_{4}$-Extrakte von Leber und Gehirn wurden gepoolt und mit $\mathrm{KOH}$ auf $\mathrm{pH} 5,0$ eingestellt. $0,2 \mathrm{ml}$ 
des Uberstandes nach Abzentrifugieren des $\mathrm{KClO}_{4}-\mathrm{Nieder}$ schlages wurden zur Analyse auf die Säule aufgebracht.

Die Bestimmungen der Konzentrationen an freien Aminosäuren erfolgten in einem Mikro-Aminosäurenanaly sator (Firma Labotron, Modell CHR-1). Eluiert wurde mit Na-Citrat-Puffer $(0,2 \mathrm{~mol} / 1 \mathrm{Citronensäure}+0,2 \mathrm{~mol} / 1 \mathrm{NaOH}, \mathrm{pH}$-Einstellung mit $\mathrm{HC} 1$ konz.) $\mathrm{pH} 3,25$ über 3 Stunden und $\mathrm{pH} 4,25$ über 11 Stunden bei $+50^{\circ} \mathrm{C}$ und einem Durchsatz von $0,5 \mathrm{ml} / \mathrm{h}$ (Ninhydrin-Zulauf 0,5 ml/h). Der photometrische Meßbereich erstreckte sich bis $5 \mathrm{nmol}$ pro Aminosäure. Photometriert wurde bei einer Wellenlänge von $570 \mathrm{~nm}$ bzw. $440 \mathrm{~nm}$ (Prolin) in $3 \mathrm{bzw} .5 \mathrm{~mm}$-Schicht. Aus den entsprechenden Eichkurven wurden die Extinktionen in $\mu \mathrm{mol}$ Aminosäure pro g Serum bzw. Organ umgerechnet.

\section{Ergebnisse}

Konzentrationen an freiem $\left[3,5-{ }^{3} \mathrm{H}\right] \mathrm{L}$-Tyrosin und $\left[{ }^{14} \mathrm{C}\right] \mathrm{L}$-Leucin in Serum und Organen

Die Konzentrationen an freiem Tyrosin und Leucin in $\mu \mathrm{mol} / \mathrm{g}$ Serum bzw. Organ, wie sie aus den radiochemischen Analysen-Ergebnissen berechnet wurden, sind der Tabelle 1 zu entnehmen.

Entsprechend der Größe der von uns als radioaktiv markiert berechneten Tyrosin-Pools (vergl. oben) erhielten die Tiere der V-Gruppe eine $200 \mathrm{fach}$ höhere Dosis an $L$-Tyrosin. Im Vergleich zur Kontrolle liegt der Serumspiegel jedoch nur um den Faktor 7 höher. In den Organextrakten finden sich dagegen 100200fach höhere Konzentrationen an freiem Tyrosin in der V-Gruppe. Diese Konzentrationsunterschiede an Tyrosin in den Organextrakten beider Gruppen sind biologisch hoch signifikant $(p<0,001)$ abgesehen vom Gehirn, wo trotz etwa 1000 fach höherer Konzentration in der V-Gruppe eine statistische Sicherung, bedingt durch die starken Streuungen, nicht möglich war. In der V-Gruppe liegen die Tyrosin-Konzentrationen in den Organen um den Faktor 5 (Milz) bis 15 (Nieren) höher als im Serum, wobei die Konzentrationsunterschiede $z$ wischen den analy sierten Organen als zufällig angesehen werden müssen. In der K-Gruppe zeigen sich dagegen in den Organen niedrigere Konzentrationen an freiem Tyrosin als im Serum. Die hohe Dosis hat sich danach vorwiegend in einer Erhöhung des Spiegels an freiem Tyrosin in den Organen, weniger aber im Serum manifestiert.

Der Serumspiegel an freiem $\left[1-{ }^{14} \mathrm{C}\right]$ Leucin zeigt in beiden Gruppen vergleichbare Werte. In den Organen Gehirn, Lunge, Milz und Nieren liegt dagegen die Konzentration an freiem Leucin in der V-Gruppe um den Faktor 1,5-3 biologisch signifikant höher $(p<0,001)$ als in den entsprechenden Organen der K-Gruppe; in der Leber zeigen sich in beiden Gruppen ausgeglichene Konzentrationen. Die Organe Lunge, Milz, Nieren und Leber weisen in der V-Gruppe höhere Spiegel an freiem $\left[1-{ }^{14} \mathrm{C}\right]$. Leucin auf als das Serum, während dies in der K-Gruppe nur bei Leber und Nieren der Fall ist.

Bei Wertung dieser Ergebnisse muß berücksichtigt werden, daß die angegebenen Konzentrationen nur für die Größe der jeweils markierten Aminosäure-Pools repräsentativ sein können. Trotz des Konzentrationsungleichgewichtes zwischen freiem Tyrosin und Leucin in den Organen der V.Gruppe wird in unseren Ergebnissen aufgezeigt, daß Leucin zu einem größeren Anteil in die Zellen der analysierten Organe permeiert sein muß als in der Kontrolle.

\section{Einbau von $\left[3,5-{ }^{3} \mathrm{H}\right] \mathrm{L}-\mathrm{Ty}$ rosin und $\left[1 \mathrm{-}^{14} \mathrm{C}\right] \mathrm{L}$-Leucin in Serum und Organ-Proteine}

Die in Serum- und Organ-Proteine eingebauten Konzentrationen an Tyrosin und Leucin in $\mu \mathrm{mol} / \mathrm{g}$, wie sie aus den spezifischen Aktivitäten der markierten Aminosäure-Pools berechnet wurden, sind in der Tabelle 2 wiedergegeben.

Die in Serum- und Organ-Proteine eingebauten Konzentrationen an Tyrosin liegen in der V-Gruppe 50 bis 100 fach gesichert höher $(p<0,001)$ als in der K-Gruppe. In Leber, Nieren und Milz liegen in der V-Gruppe 2 bis 3 fach höhere Einbauraten vor als in Lunge und Gehirn. Legt man ausgehend von den Daten der Tabelle 2 eine mittlere Einbaurate von $5 \mu \mathrm{mol}$ Tyrosin/g Tier zugrunde, so entspricht dies einer Einbaurate in Proteine von $15 \%$ der Dosis $(=45 \mathrm{mg} /$ Tier $)$ in der VGruppe. In der K-Gruppe wurde etwa ein Anteil von $30 \%$ des als markiert angenommenen Tyrosin-Pools $(=450 \mu \mathrm{g} /$ Tier $)$ in den 7 Stunden Versuchsdauer zur Protein-Synthese genutzt.

Der Einbau von $\left[1{ }^{14} \mathrm{C}\right]$ Leucin aus dem 2 Stunden vor Tötung der Tiere markierten Pool in Proteine liegt bei beiden Gruppen in vergleichbaren Größenordnungen, wobei lediglich die Serumproteine der V-Gruppe gegenüber denen der K-Gruppe eine um den Faktor 1,5 gesichert höher liegende Einbaurate an Leucin aufzeigen $(\mathrm{p}<0,001)$.

Aus diesen Ergebnissen läßt sich ableiten, daß trotz erhöhter Spiegel an freiem Tyrosin in den Organen kein negativer Einfluß auf die Proteinsyntheserate durch Hemmung des Transportes essentieller Aminosäuren in die Zellen der analysierten Organe vorgelegen hat. Die gesichert höher liegenden Konzentrationen an freiem $\left[1{ }^{14} \mathrm{C}\right]$ Leucin in den Organen der V-Gruppe im Vergleich zur Kontrolle lassen den Schluß zu, daß die Erhöhung des Tyrosinspiegels sogar eine Erhöhung der Permeation essentieller Aminosäuren in die Zellen zur Folge hatte. Der erhöhte Einbau von $\left[1^{14} \mathrm{C}\right]$ Leucin in die Serum-Proteine der V-Gruppe könnte für eine Stimulierung der Proteinsynthese in der Leber sprechen.

\section{Abbau von $\left[3,5-{ }^{3} \mathrm{H}\right] \mathrm{L}$-Tyrosin zu Stoffwechselendpro- dukten}

Bei der Tritiierung des Tyrosins am $\mathrm{C}_{3}$ - und am $\mathrm{C}_{5}$ Atom des Phenolrings entspricht die Bildungsrate an ${ }^{3} \mathrm{H}_{2} \mathrm{O}$ dem Abbau der Aminosäure zu Stoffwechselendprodukten. Ein Isotopie-Effekt kann in diesem Zusammenhang als vernachlässigbar gering angesehen werden. 
Tab. 1. Spezifische Konzentrationen an freiem $\left[{ }^{3} \mathrm{H}\right]$ Tyrosin und $\left[{ }^{14} \mathrm{C}\right]$ Leucin in Serum und Organen ( $\mu$ mol/g Organ) bei der Ratte nach i. p. Applikation von $5 \mathrm{mg}\left[{ }^{3} \mathrm{H}\right]$ Tyrosin/g Körpergewicht (Versuchsgruppe; $\left.n=9\right)$ bzw. $25 \mu \mathrm{g} \cdot\left[{ }^{3} \mathrm{H}\right]$ Tyrosin/g Körpergewicht (Kontrollgruppe; $n=10$ ) und i. v. Applikation von $\left.\left.20 \mu \mathrm{g}\right|^{14} \mathrm{C}\right]$ Leucin/g Körpergewicht. Mittelwerte ( \pm Standardabweichung) und Signifikanz der Unterschiede beider Gruppen.

\begin{tabular}{|c|c|c|c|c|c|c|c|c|}
\hline \multirow{2}{*}{ Organ } & \multicolumn{4}{|c|}{$\mu \mathrm{mol}\left[{ }^{3} \mathrm{H}\right]$ Tyrosin/g } & \multicolumn{4}{|c|}{$\mu \mathrm{mol}\left[{ }^{14} \mathrm{C}\right]$ Leucin $/ \mathrm{g}$} \\
\hline & $\begin{array}{l}\text { Versuchs- } \\
\text { gruppe }\end{array}$ & $\begin{array}{l}\text { Kontroll- } \\
\text { gruppe }\end{array}$ & $\mathbf{t}$ & $\mathbf{p}$ & $\begin{array}{l}\text { Versuchs- } \\
\text { gruppe }\end{array}$ & $\begin{array}{l}\text { Kontroll- } \\
\text { gruppe }\end{array}$ & $\mathbf{t}$ & $\mathbf{p}$ \\
\hline Serum & 0,1969 & 0,0287 & & & 0,0074 & $0 ; 0065$ & & \\
\hline Gehirn & $\begin{array}{c}1,15 \\
(1,524)\end{array}$ & $\begin{array}{l}0,001 \\
(0,00029)\end{array}$ & 2,38 & 0,05 & $\begin{array}{c}0,003 \\
(0,0008)\end{array}$ & $\begin{array}{c}0,0019 \\
(0,0006)\end{array}$ & 3,48 & 0,001 \\
\hline Lunge & $\begin{array}{l}1,58 \\
(1,221)\end{array}$ & $\begin{array}{l}0,005 \\
(0,0015)\end{array}$ & 4,08 & 0,001 & $\begin{array}{c}0,013 \\
(0,003)\end{array}$ & $\begin{array}{c}0,0039 \\
(0,0017)\end{array}$ & 8,34 & 0,001 \\
\hline Milz & $\begin{array}{c}0,92 \\
(1,017)\end{array}$ & $\begin{array}{c}0,012 \\
(0,0057)\end{array}$ & 2,82 & 0,01 & $\begin{array}{c}0,013 \\
(0,004)\end{array}$ & $\begin{array}{c}0,0040 \\
(0,0011)\end{array}$ & 6,86 & 0,001 \\
\hline Nieren & $\begin{array}{c}2,91 \\
(1,793)\end{array}$ & $\begin{array}{c}0,009 \\
(0,0017)\end{array}$ & 5,12 & 0,001 & $\begin{array}{c}0,032 \\
(0,008)\end{array}$ & $\begin{array}{c}0,0108 \\
(0,0041)\end{array}$ & 7,46 & 0,001 \\
\hline Leber & $\begin{array}{c}1,62 \\
(0,442)\end{array}$ & $\begin{array}{c}0,011 \\
(0,0087)\end{array}$ & 11,51 & 0,001 & $\begin{array}{c}0,013 \\
(0,002)\end{array}$ & $\begin{array}{c}0,0120 \\
(0,0117)\end{array}$ & 0,26 & 0,7 \\
\hline
\end{tabular}

Tab. 2. Einbau von $\left[{ }^{3} \mathrm{H}\right]$ Tyrosin und $\left[{ }^{14} \mathrm{C}\right]$ Leucin in Serum- und Organproteine $(\mu \mathrm{mol} / \mathrm{g}$ Organ) bei der Ratte nach i. p. Applikation von $\left.5 \mathrm{mg} \mathrm{[}{ }^{3} \mathrm{H}\right]$ Tyrosin/g Körpergewicht (Versuchsgruppe; $n=9$ ) bzw. $\left.25 \mu \mathrm{g} \mathrm{[}{ }^{3} \mathrm{H}\right]$ Tyrosin/g Körpergewicht (Kontrollgruppe; $\mathrm{n}=10)$ und i. v. Applikation von $20 \mu \mathrm{g}\left[{ }^{14} \mathrm{C}\right]$ Leucin/g Körpergewicht.

Mittelwerte ( \pm Standardabweichung) und Signifikanz der Unterschiede zwischen beiden Gruppen.

\begin{tabular}{|c|c|c|c|c|c|c|c|c|}
\hline \multirow{2}{*}{ Organ } & \multicolumn{4}{|c|}{$\mu$ mol $\left[{ }^{3} \mathrm{H}\right]$ Tyrosin in Proteinen/g Organ } & \multicolumn{4}{|c|}{$\mu$ mol $\left[{ }^{14} \mathrm{C}\right]$ Leucin in Proteinen/g Organ } \\
\hline & $\begin{array}{l}\text { Versuchs- } \\
\text { gruppe }\end{array}$ & $\begin{array}{l}\text { Kontroll- } \\
\text { gruppe }\end{array}$ & $t$ & p & $\begin{array}{l}\text { Versuchs- } \\
\text { gruppe }\end{array}$ & $\begin{array}{l}\text { Kontroll- } \\
\text { gruppe }\end{array}$ & $\mathbf{t}$ & $\mathbf{p}$ \\
\hline Serum & $\begin{array}{l}6,815 \\
(1,5065)\end{array}$ & $\begin{array}{l}0,073 \\
(0,0001)\end{array}$ & 14,152 & 0,001 & $\begin{array}{c}0,069 \\
(0,008)\end{array}$ & $\begin{array}{c}0,046 \\
(0,0059)\end{array}$ & 7,317 & 0,001 \\
\hline Gehirn & $\begin{array}{c}2,018 \\
(0,3966)\end{array}$ & $\begin{array}{c}0,018 \\
(0,0067)\end{array}$ & 15,944 & 0,001 & $\begin{array}{c}0,0192 \\
(0,005)\end{array}$ & $\begin{array}{c}0,019 \\
(0,0018)\end{array}$ & 0,119 & 0,05 \\
\hline Lunge & $\begin{array}{c}2,799 \\
(1,091)\end{array}$ & $\begin{array}{c}0,053 \\
(0,0158)\end{array}$ & 7,958 & 0,001 & $\begin{array}{c}0,0313 \\
(0,009)\end{array}$ & $\begin{array}{c}0,044 \\
(0,0123)\end{array}$ & 2,63 & 0,02 \\
\hline Milz & $\begin{array}{c}7,624 \\
(1,906)\end{array}$ & $\begin{array}{c}0,108 \\
(0,0243)\end{array}$ & 12,470 & 0,001 & $\begin{array}{c}0,067 \\
(0,017)\end{array}$ & $\begin{array}{c}0,083 \\
(0,020)\end{array}$ & 1,93 & 0,05 \\
\hline Nieren & $\begin{array}{c}5,974 \\
(3,619)\end{array}$ & $\begin{array}{c}0,069 \\
(0,0225)\end{array}$ & 5,16 & 0,001 & $\begin{array}{c}0,061 \\
(0,006)\end{array}$ & $\begin{array}{c}0,054 \\
(0,0062)\end{array}$ & 2,86 & 0,02 \\
\hline Leber & $\begin{array}{c}6,174 \\
(2,020)\end{array}$ & $\begin{array}{l}0,048 \\
(0,0221)\end{array}$ & 9,59 & 0,001 & $\begin{array}{c}0,064 \\
(0,019)\end{array}$ & $\begin{array}{c}0,034 \\
(0,0147)\end{array}$ & 2,28 & 0,05 \\
\hline
\end{tabular}

In der V-Gruppe analysierten wir $42,2( \pm 3,7) \%$ der Radioaktivitätsdosis als ${ }^{3} \mathrm{H}_{2} \mathrm{O}$, was bei einer Dosis von $300 \mathrm{mg}$ Tyrosin/Tier einer Abbaurate von $126 \mathrm{mg}$ Tyrosin entspricht. Bei der K-Gruppe wurden im gleichen Versuchszeitraum 3,1 $( \pm 0,22) \%$ der Radioaktivitätsdosis als ${ }^{3} \mathrm{H}_{2} \mathrm{O}$ nachgewiesen, was bei einer Größe des markierten Pools von $1,5 \mathrm{mg}$ Tyrosin/Tier einem Abbau von $46,5 \mu \mathrm{g}$ entspricht.

Diese hohe Tyrosin-Abbaurate in der V-Gruppe erklärt, daß trotz hoher Dosierung nur relativ niedrige Konzentrationen an freiem und utilisiertem Tyrosin nachgewiesen wurden.

\section{Konzentrationen an freien Aminosäuren in Serum, Gehirn und Leber}

Die Konzentrationen an freien Aminosäuren in $\mu \mathrm{mol} / \mathrm{g}$ Serum, Gehirn und Leber nach säulenchromatographischer Analyse zeigt die Tabelle 3 .

In der V-Gruppe liegen die Konżentrationen aller Aminosäuren im Serum auffallig niedriger als in der Kontrolle mit Ausnahme von Tyrosin, das etwa um den Faktor 3 höher liegt. In Gehirn und Leber fanden wir dagegen in der V-Gruppe deutlich höhere Konzentrationen an Aminosäuren als in der K-Gruppe, abgesehen 
Tab. 3. Spezifische Konzentrationen an freien Aminosäuren ( $\mu \mathrm{mol} / \mathrm{g})$ in Serum, Gehirn und Leber der Ratte nach i. p. Applikation von $5 \mathrm{mg}$ Tyrosin/g Körpergewicht (Versuchsgruppe) im Vergleich zu einer Kontrollgruppe.

\begin{tabular}{|c|c|c|c|c|c|c|}
\hline \multirow[t]{2}{*}{ Aminosäure } & \multicolumn{2}{|c|}{ Serum } & \multicolumn{2}{|c|}{ Gehirn } & \multicolumn{2}{|c|}{ Leber } \\
\hline & Versuch & Kontrolle & Versuch & Kontrolle & Versuch & Kontrolle \\
\hline Asparaginsäure & 0,16 & 0,44 & 0,54 & 0,44 & 0,24 & 0,11 \\
\hline Threonin & 0,29 & 0,67 & 0,10 & 0,08 & 0,20 & 0,13 \\
\hline Serin & 0,40 & 0,47 & 0,28 & 0,48 & 0,25 & 0,14 \\
\hline Prolin & 0,15 & 0,41 & 0,12 & - & 0,23 & - \\
\hline Glutaminsäure & 0,28 & 0,37 & 0,35 & 0,66 & 0,49 & 0,55 \\
\hline Glycin & 0,36 & 0,57 & 0,23 & 0,19 & 0,79 & 0,56 \\
\hline Alanin & 0,35 & 1,14 & 0,25 & 0,19 & 1,64 & 0,79 \\
\hline Cystein & 0,02 & 0,12 & 0,02 & 0,03 & 0,07 & 0,08 \\
\hline Valin & 0,06 & 0,29 & 0,08 & 0,04 & 0,20 & 0,09 \\
\hline Methionin & 0,08 & 0,08 & 0,04 & 0,08 & 0,07 & 0,03 \\
\hline Isoleucin & 0,08 & 0,14 & 0,05 & 0,02 & 0,10 & 0,07 \\
\hline Leucin & 0,14 & 0,27 & 0,10 & 0,05 & 0,24 & 0,14 \\
\hline Tyrosin & 0,26 & 0,08 & 0,06 & 0,02 & 0,09 & 0,05 \\
\hline Phenylalanin & 0,09 & 0,14 & 0,06 & 0,03 & 0,22 & 0,14 \\
\hline
\end{tabular}

von Serin, Glutaminsäure und Cystein in Leber und Gehirn und Methionin im Gehirn, die in der Kontrolle höhere Konzentrationen aufwiesen. Trotz der hohen Dosis liegt der Spiegel an freiem Tyrosin in Serum und Gehirn nur 3 fach, in der Leber nur 2 fach höher als in der K-Gruppe, wobei in der V-Gruppe die Konzentrationen im Gehirn nur $1 / 4$ und in der Leber nur $1 / 3$ der Serumkonzentration betragen.

Diese Ergebnisse deuten in ihrer sicheren Tendenz darauf hin, daß die „Verarmung" des Serums an Aminosäuren in der V-Gruppe auf eine vermehrte Permeation in die Zellen der Organe bzw. Gewebe zurückgeführt werden kann. Nach unseren Befunden kann demnach eine Hemmung des Aminosäuren-Transportes in die Zellen durch erhöhte Konzentrationen an Tyrosin ausgeschlossen werden. Vielmehr scheint ein Ausgleich der relativ geringen Aminosäuren-Imbalance in den Zellen stattgefunden zu haben. Das vergleichsweise hohe Konzentrationsgefalle von Serum zu Organen könnte vielmehr im Sinne einer Transporthemmung des Tyrosins gedeutet werden.

\section{Diskussion}

Ein wesentliches Ergebnis unserer Untersuchungen ist darin zu sehen, daß trotz einer ausgesprochen hohen Dosis von $5 \mathrm{~g}$ Tyrosin/kg Körpergewicht eine hypertyrosinämische Stoffwechsellage mit einer Erhöhung des Serum-Spiegels auf ein Vielfaches der Norm und damit eine mögliche Aminosäuren-Imbalance mit ihrer Auswirkung auf die Protein-Synthese nicht zu erreichen war. Weder Tyrosin selbst noch intermediäre Stoffwechselprodukte beeinflußten die Permeation essentieller Aminosäuren in die Zellen der von uns analysierten Organe und damit die Utilisation von Leucin in der Protein-Synthese. In unserem Versuch reagierten die Ratten auf die hohe intraperitoneale Tyrosin-Dosis mit einer Aktivierung des Stoffwechsels im Sinne einer hohen Abbaurate zu Endprodukten.
Die Ergebnisse der radiochemischen Analysen erlauben die Aufstellung einer Bilanz, nach der in den 7 Stunden Versuchsdauer $42 \%$ der Dosis an Tyrosin zu Endprodukten verstoffwechselt, $15 \%$ in der Protein-Synthese utilisiert und 5\% in Form der freien Aminosäure im Organismus der Tiere nachgewiesen wurde. Bei der hohen Funktionsleistung der Rattenniere dürften im Versuchszeitraum mindestens 10\% der Dosis in Form der Aminosäure bzw. ihrer Stoffwechselprodukte renal eliminiert worden sein (da unsere Untersuchungen auf die Auswirkungen einer Aminosäuren-Imbalance bei der Hypertyrosinämie ausgerichtet waren, wurde auf eine Analyse der Ausscheidungen verzichtet). Wir dürfen also bei unseren Untersuchungen mit einer Mindest-Resorptionsrate von $70 \%$ aus dem Intraperitonealraum rechnen; bei Tötung der Tiere war Tyrosin im Intraperitonealraum noch makroskopisch sichtbar nachzuweisen. Danach hat ein ausreichender Depoteffekt, wie er auch in der Literatur (14) beschrieben wird, über den gesamten Untersuchungszeitraum vorgelegen, der bei einem entsprechenden Konzentrationsgefälle zu einer laufenden Einschleusung von Tyrosin in den Kreislauf, und damit auch während der 2 Stunden nach Leucin-Applikation bis zur Tötung der Tiere, geführt hat.

Beim Vergleich der radiochemischen und der säulenchromatographischen Analysen-Ergebnisse zeigen sich nach beiden Verfahren im Serum der Versuchsgruppe etwa gleiche Tyrosin-Konzentrationen. In Leber und Gehirn wurden dagegen radiochemisch etwa $20 \mathrm{fach}$ höhere Konzentrationen an Tyrosin ermittelt als nach dem säulenchromatographischen Verfahren. Diese unterschiedlichen Konzentrationswerte müssen auf die über die ${ }^{3} \mathrm{H}$-Radioaktivität miterfaßten intermediären Stoffwechselprodukte des Tyrosins (Fumarat, Acetoacetat, Homogentisinat) zurückgeführt werden. Dieser relativ hohe Anteil an Stoffwechselprodukten in den analysierten Organen, nicht aber im Serum, deutet daraufhin, daß Tyrosin in diesen Organen metabo- 
lisiert wurde. Eine Anreicherung dieser Intermediärprodukte in den Organen über den Blutweg ist unwahrscheinlich, da diese hydrophilen Substanzen von der Niere rasch eliminiert werden. Danach muß es sich bei diesen Stoffwechselprodukten in den Zellen der Organe um kurzlebige Metabolite mit einem hohen turnover handeln, denen aber ebenfalls kุein störender Einfluß auf den Transport essentieller Aminosäuren und damit die Protein-Synthese zugeschrieben werden kann.

Die Ergebnisse dieser Untersuchungen dürften die Ratte als geeignetes Versuchstier zum Studium der Auswirkung einer experimentellen Hypertyrosinämie in Frage stellen. Nach Henze et al (2) spielt das Alter der Versuchstiere insofern eine Rolle, als eine experimentelle Hypertyrosinämie sich bei Ratten älter als 20 Tage nur bedingt auswirkt. Bei jüngeren Ratten scheint die durch Aktivierung der Tyrosin-OxoglutaratAminotransferase bedingte Anreicherung von Hy- droxyphenylbrenztraubensäure zu einer Hemmung der Hydroxyphenylpyruvat-Hydroxylase zu führen. Der resultierende Anstau von Hydroxyphenylpyruvat kann damit eher zu der gewünschten Erhöhung des Spiegels an Tyrosin füren. Die von uns angewendete Methodik erforderte jedoch Mindestmengen an Probenmaterial, wodurch wir auf Rattèn mit entsprechenden Körpergewichten angewiesen waren. Die Uberprüfung der Auswirkung einer experimentellen Hypertyrosinämie im Sinne einer Aminosäuren-Imbalance und einer dadurch gestörțen Proteinsynthese bei jungen Ratten bleibt entsprechenden Untersuchungen vorbehalten.

\section{Danksagung}

Fräulein M. Clausnitzer, Frau R. Thome, Frau G. Wenske und Herrn $H$. Kreyling sei an dieser Stelle für ihre wertvolle Hilfe bei der Durchführung der Untersuchungen gedankt.

\section{Literatur}

1. Menkes, J. H. \& Avery M. E. (1963), Bull. Johns Hopkins Hosp. 113, 301-219

2. Henze, H. \& Bremer, H. J. (1969) Mschr. Kinderheilk. 117, 433-436

3.Menkes, J. H., Welcher, D. W., Levi, H. S., Dallas, J. \& Gretsky, N. E. (1972), Pediatrics 49, 218-224

4. Menkes, J. H., Welcher D. W., Levi, H. S., Stern, E. R., Dallas, J. \& Gretsky, N. E. (1971) Pediat. Res. 5, 372-433

5. Menkes, J. H. \& Jervis, G. A. (1961) Pediatrics 28, 399409

6. Snyderman, S. E., Holt jr., L. E. Morton, P. M. Rostman, E \& Phansalkar, S. V. (1968), Pediat. Res. 2, 131-144

7. Linneweh, F. \& Ehrlich, M. (1962), Klin. Wochenschr. 40, $225-226$
8. Mckean, C. M., Boggs, D. E. \& Peterson, N. A. (1968) J. Neurochem. 15, 235-241

9. Peterson, N. A. \& McKeam, C. M. (1969) J. Neurochem. $16,1211-1217$

10. Vahvelainen, M. Z. \& Oja, S. S. (1969) Brain Res. 13, 227-233

11. Lindroos, O. F. C. \& Oja, S. S. (1971) Exp. Brain, Res. 14,48

12. Neame, K. D. (1961), Nature (Lond.), 192, 173-174

13. Guroff, G. \& Udenfriend, S. (1962), J. Biol. Chem. 237, 803-806

14. Chirigos, M. A., Greengard \& Udenfriend, S. (1960) J. Biol. Chem. 235, 2075-2079

Professor Dr. E. Schaumlöffel Klinik und Poliklinik für Nuklearmedizin im Radiologiezentrum der Philipps-Universität 355 Marburg (Lahn) Lahnstraße 4 a 